\title{
How do the clinical findings in patients with pericardial effusions influence the success of aspiration?
}

\author{
J P Cooper, R M Oliver, P Currie, J M Walker, R H Swanton
}

\begin{abstract}
Objective-To identify features associated with success or failure of aspiration of pericardial effusion.

Method-A retrospective analysis of 36 drainage procedures in 30 patients with pericardial effusion was performed using patient records and echocardiograms.

Results-Unsuccessful aspiration was associated with pericardial loculation but not with the seniority of the operator or the size and position of the effusion. Pericardiocentesis relieved symptoms of breathlessness in 21 of 26 patients who had a pericardial effusion suspected of causing dyspnoea. These 21 patients had few clinical or echocardiographic signs of classic tamponade.

Conclusion-The paucity of abnormal physical or echocardiographic signs of tamponade in breathless patients with pericardial effusion does not exclude symptomatic benefit being derived from pericardiocentesis. Pericardial aspiration is safe in appropriate hands, although aspiration of loculated effusions may not be as successful as aspiration of non-loculated effusions.
\end{abstract}

(Br Heart f 1995;73:351-354)

Keywords: pericardial effusion, pericardiocentesis, cardiac tamponade

The optimal time to drain a pericardial effusion is often difficult to determine. If symptoms and signs of haemodynamic compromise are severe there is little doubt that immediate drainage is warranted. More difficult to assess is the need for drainage in those patients who may have few symptoms or signs as in some the effusion may resolve without aspiration.

A retrospective survey of 36 drainage procedures in 30 patients with pericardial effusion was performed to identify which patient characteristics are associated with successful pericardial aspiration.

\section{Patients and methods}

Patients $(n=30)$ with pericardial effusions requiring drainage between 1989 and 1993 were identified from echocardiograms and corresponding patient records. The patients' hospital records and electrocardiograms (ECGs) were examined by one cardiologist (JPC) and their echocardiograms were examined independently by two experienced cardi- ologists (JPC and RMO). If they disagreed, the echocardiogram was reviewed together and a consensus reached and if this was not possible an independent cardiologist was consulted. The following features were noted:

(a) presenting symptoms;

(b) relief of symptoms by aspiration;

(c) physical signs of tamponade: tachycardia (ventricular rate $>100$ beats $/ \mathrm{min}$ ), hypotension (systolic blood pressure $<100 \mathrm{~mm} \mathrm{Hg}$ ), pulsus paradoxus $(>10 \mathrm{~mm} \mathrm{Hg}$ change in peak systolic pressure on inspiration compared with that on expiration), positive Kussmaul's sign, and elevation of the jugular venous pressure ( $>3 \mathrm{~cm}$ above sternal angle);

(d) ECG changes (small complexes: QRS < $0.5 \mathrm{mV}$ limb leads and $<1.0 \mathrm{mV}$ chest leads, electrical alternans: successive QRS complexes alternating higher then lower amplitude);

(e) echocardiographic evidence of chamber collapse and its severity, which was scored as $0=$ absent, $1=$ mild, $2=$ moderate, $3=$ severe. Chamber collapse was defined as (i) right atrial collapse: at least $30 \%$ of the right atrial wall inverted during late diastole, early systole, or both; (ii) left atrial collapse: any portion of the left atrial wall inverted during late diastole/early systole; (iii) right/left ventricular diastolic collapse: inward motion of the right/left ventricular wall in early diastole persisting after mitral valve opening;

$(f)$ the maximum width of the effusion measured as a line drawn perpendicular to the wall of the heart;

$(g)$ the position of the effusion (anterior/ posterior/lateral/circumferential);

(h) the presence of loculation;

(i) the size of effusion (small/medium/ large);

(j) the volume of fluid drained; and

$(k)$ aetiology of the effusion.

\section{STATISTICAL METHODS}

Observer correlation of chamber collapse is presented using Pearson's correlation coefficient. Clinical features associated with successful or unsuccessful aspiration were analysed using the $\chi^{2}$ test.

\section{Results}

PATIENT CHARACTERISTICS

Thirty patients ( 12 female, 18 male, age range 9-81 years) underwent pericardiocentesis. Four patients underwent two aspirations and one patient underwent three. The cause of the effusions were postcardiac surgery $(14(47 \%)$ of 30 patients), malignancy (eight $(27 \%)$ of 
Figure 1 Parasternal long axis view of the only pericardial effusion that was surgically drained without first attempting pericardiocentesis in $(A)$ systole and $(B)$ diastole. systole and (B) diastole.
Note left ventricular diastolic collapse (lvdc) of the inferior wall of the lefi ventricle caused by the pericardial effusion (PE) This patient had cardiac tamponade. $L V$, left ventricle; $L A$, left atrium
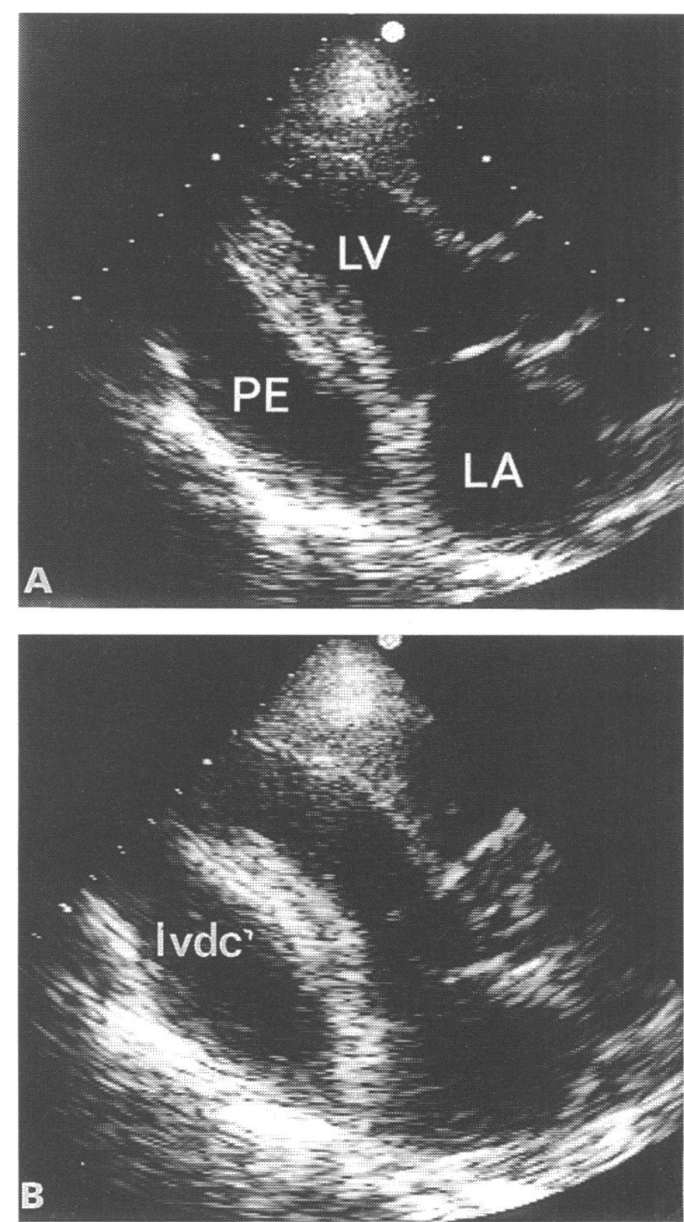

30 ), unknown (six (20\%) of 30), and bacterial pericardial infection (one (3\%) of 30). One patient $(3 \%)$ had a bacterial pericardial infection after cardiac surgery.

The most common presenting symptoms were breathlessness ( $26(87 \%)$ of 30 patients) and chest pain (six (20\%) of 30), although fever (five $(17 \%)$ of 30 ), cough (two $(7 \%)$ of $30)$, lethargy (1 (3\%) of 30), and palpitations (one $(3 \%)$ of 30 ) were also described. All 26

Table 1 Physical signs of patients in whom pericardial drainage was successful $(n=21)$ or unsuccessful $(n=5)$ at relieving symptoms of breathlessness

\begin{tabular}{|c|c|c|c|}
\hline \multirow[b]{2}{*}{ Sign } & \multicolumn{2}{|l|}{ Tamponade } & \multirow{2}{*}{$\begin{array}{l}\text { Physical sign } \\
\text { not recorded }\end{array}$} \\
\hline & Relieved $(n=21)$ & Not relieved $(n=5)$ & \\
\hline $\begin{array}{l}\text { Tachycardia } \\
\quad(>100 \text { beats } / \mathrm{min})\end{array}$ & $13 / 20(65)$ & $2 / 5(40)$ & 1 \\
\hline $\begin{array}{l}\text { Pulsus paradoxus } \\
(>10 \mathrm{~mm} \mathrm{Hg})\end{array}$ & $10 / 18(56)$ & $2 / 5(40)$ & 3 \\
\hline $\begin{array}{l}\text { Hypotension } \\
(<100 \mathrm{~mm} \mathrm{Hg})\end{array}$ & $6 / 20(30)$ & $1 / 5(20)$ & 1 \\
\hline $\begin{array}{l}\text { Increased jugular } \\
\text { venous pressure } \\
(>3 \mathrm{~cm})\end{array}$ & $10 / 19(53)$ & $2 / 4(50)$ & 3 \\
\hline Positive Kussmaul's sign & $5 / 19(26)$ & $1 / 5(20)$ & 2 \\
\hline
\end{tabular}

Values in parentheses are percentages.

Table 2 Echocardiographic signs of chamber collapse in patients in whom pericardial drainage was successful $(n=21)$ or unsuccessful $(n=5)$ at relieving symptoms of breathlessness

\begin{tabular}{llll}
\hline & \multicolumn{2}{l}{ Tamponade } & Chamber collapse \\
\cline { 2 - 3 } Chamber collapse & Relieved $(n=21)$ & Not relieved $(n=5)$ & \\
\hline Right ventricle & $13 / 21(62)$ & $4 / 5(80)$ & 0 \\
Left ventricle & $1 / 20(5)$ & $0 / 5$ & 1 \\
Right atrium & $8 / 19(42)$ & $1 / 4(25)$ & 3 \\
Left atrium & $3 / 21(19)$ & $1 / 4(25)$ & 1 \\
\hline
\end{tabular}

Values in parentheses are percentages. patients suspected of having tamponade were breathless; the other four underwent pericardial aspiration because of the size of the effusion (two), suspected pericardial infection (one) and the presence of a pericardio-skin fistula after cardiac surgery (one). The median (range) time from onset of symptoms to time of drainage was 10 (1-300) days and from time of surgery to drainage was 29 (5-365) days. All patients underwent pericardiocentesis from the subxiphisternal route, apart from one who had a loculated effusion causing isolated left ventricular collapse, in whom surgical drainage was performed (fig 1).

\section{OBSERVER CORRELATION}

There was good correlation between the two observers as to the degree of left atrial collapse $(r=0.81, \mathrm{n}=26)$, right atrial collapse $(r=0 \cdot 69, \mathrm{n}=32)$, right ventricular collapse $(r=0.87, \mathrm{n}=36)$, and left ventricular collapse $(r=0 \cdot 84, \mathrm{n}=36)$. At least one cardiologist thought that there were inadequate views of the heart to detect left atrial collapse in eight $(22 \%)$ cases and right atrial collapse in four $(11 \%)$. The right ventricle and left ventricle were seen adequately in all patients to assess collapse in these chambers.

SIGNS OF PERICARDIAL TAMPONADE

The initial pericardiocentesis relieved symptoms of breathlessness in 21 of the 26 breathless patients all of whom were suspected of having tamponade. Pericardial fluid was not aspirated in two of three breathless patients who derived no benefit from initial pericardiocentesis and anaemia was considered as a possible cause of breathlessness in one. Two patients had only a mild improvement in breathlessness. Tables 1 and 2 give the frequency of abnormal clinical and echocardiographic signs present before drainage in those patients in whom symptoms of breathlessness were relieved by this procedure. Three (16\%) of 19 patients in whom pericardial drainage relieved symptoms, had no abnormal physical signs (tachycardia, pulsus paradoxus, hypotension, increased jugular venous pressure, or positive Kussmaul's sign) and only one of these abnormal signs was present in four $(21 \%)$ of 19 . Data of the two remaining patients in this group were missing making it impossible to assess whether or not a sign was absent. Features of chamber collapse did not occur in six $(29 \%)$ of 21 patients and no clinical signs of a pericardial effusion or echocardiographic evidence of chamber collapse occurred in three $(16 \%)$ of 19 . The effusions were noted to be large in $12(57 \%)$ of 21 patients, moderate in eight $(38 \%)$ and small in one $(5 \%)$. Small ECG complexes were present in four $(22 \%)$ of 18 patients one of whom had electrical alternans.

DETERMINANTS OF SUCCESSFUL PERICARDIAL ASPIRATION

Successful aspiration was defined as any volume of pericardial fluid above that required to relieve dyspnoea in the 26 aspiration procedures performed in patients with suspected tamponade. All five aspirates with 


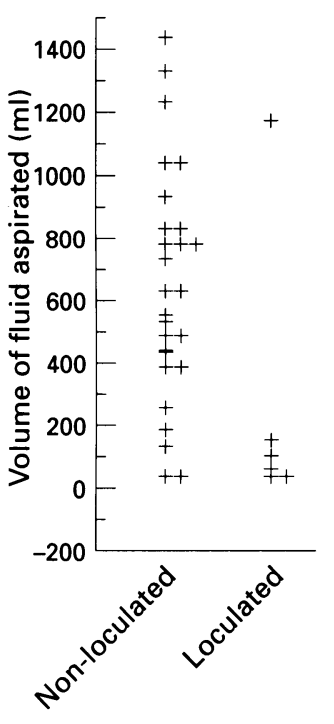

Figure 2 Volume of fluid aspirated in loculated and non-loculated effusions.
Table 3 Echocardiographic features associated with successful or unsuccessful pericardiocentesis

\begin{tabular}{|c|c|c|}
\hline & $\begin{array}{l}\text { Successful }(n=26) \\
\text { pericardiocentesis }\end{array}$ & $\begin{array}{l}\text { Failured }(n=8) \\
\text { pericardiocentesis }\end{array}$ \\
\hline Loculation present & $1(17)$ & $5(83)^{\star}$ \\
\hline $\begin{array}{l}\text { Mean (SE) effusion } \\
\text { width }(\mathrm{cm})\end{array}$ & $2.9(0 \cdot 2)$ & $2 \cdot 8(0 \cdot 2)$ \\
\hline \multicolumn{3}{|l|}{ Effusion position } \\
\hline Posterior & $3(50)$ & $3(50)$ \\
\hline Circumferential & $21(81)$ & $5(19)$ \\
\hline Anterior & $2(100)$ & 0 \\
\hline \multicolumn{3}{|l|}{ Operator } \\
\hline Consultant & $5(71)$ & $2(29)$ \\
\hline Senior registrar & $7(88)$ & $1(12)$ \\
\hline Registrar & $13(74)$ & $1(26)$ \\
\hline
\end{tabular}

volumes $<120 \mathrm{ml}$ (mean (range) $66(0-120)$ $\mathrm{ml}$ ) failed to relieve dyspnoea, while all 21 aspirates with volumes $>120 \mathrm{ml}$ (mean (range) $692(150-1400) \mathrm{ml}$ ) relieved dyspnoea. Successful and unsuccessful aspirations were therefore defined as aspirations of greater or less than $120 \mathrm{ml}$ respectively.

None of the classical clinical signs associated with tamponade was consistently associated with success or failure of aspiration in breathless patients (table 1). When all aspiration procedures were analysed, neither the position of the effusion, nor the maximum effusion width influenced whether drainage was successful. The presence of loculation, however, was significantly associated with unsuccessful aspiration (fig 2 and table 3), although it was possible to drain $1140 \mathrm{ml}$ of fluid from one effusion which was considered to be loculated. One effusion while initially non-loculated and successfully aspirated, recurred and became loculated over a period of three weeks and could not be successfully aspirated. Successful procedures were less painful (data not shown) and not influenced by the seniority of the operator. Despite the high rate of unsuccessful aspiration (eight $(24 \%)$ of 34 procedures) there were no major complications from pericardiocentesis, although perforation of the right ventricle was noted in two cases.

\section{Discussion}

The most common reason for aspirating a pericardial effusion is because of the development of cardiac tamponade. Starling ${ }^{1}$ defined cardiac tamponade as compression of the heart by fluid within the pericardial sac impairing diastolic filling of the ventricles. Other clinical definitions have arisen which have embraced the characteristic physical signs of tamponade with their relief by pericardiocentesis, although acknowledging that certain physical signs are not invariably present. ${ }^{2}$ We have defined tamponade to mean a pericardial effusion causing dyspnoea which is relieved by aspiration of the effusion. A patient with tamponade typically develops a group of clinical findings: dyspnoea, tachycardia, pulsus paradox, hypotension, increased jugular venous pressure and a positive Kussmaul's sign. The ECG may show evidence of small complexes and electrical alternans and the chest $x$ ray cardiomegaly. The echocardiogram may demonstrate a pericardial effusion causing atrial or ventricular col- lapse depending on the pressure and location of fluid in the pericardial space. ${ }^{3-9}$ Little is known about the frequency of these clinical features in relation to the likelihood of symptomatic relief being achieved by pericardiocentesis. Furthermore, other clinical features affecting the likelihood of pericardial aspiration being successful have not previously been assessed. We therefore analysed 36 drainage procedures performed in 30 patients with pericardial effusion in an attempt to identify patient characteristics which may predict the likelihood of successful pericardial aspiration.

CLINICAL SIGNS IN PATIENTS WITH SUSPECTED PERICARDIAL TAMPONADE

All 26 patients with symptomatic pericardial effusion complained of dyspnoea as their primary symptom. Of these, 21 improved with pericardiocentesis suggesting ventricular diastolic filling was impaired resulting in tamponade. Clinical signs of tamponade, however, were frequently not seen in this group in which successful pericardiocentesis had occurred; three (16\%) of 19 patients had no clinical signs of tamponade and four $(21 \%)$ of 19 only one. The more specific the clinical sign for cardiac tamponade the less frequently it was observed: tachycardia was the most common physical sign of a symptomatic effusion but was present only in $65 \%$ of patients compared with $74-77 \%$ in other series. ${ }^{210}$ Pulsus paradoxus was present only in $56 \%$ of patients compared with $36-77 \%$ in other series. ${ }^{2310}$ Increased jugular venous pressure was present in $53 \%$ of patients compared with $74-100 \%$ in other series. ${ }^{210}$ Hypotension occurred in $30 \%$ of patients compared with $14-36 \%$ in other series, ${ }^{210}$ while Kussmaul's sign, the most specific sign of pericardial disease, was present only in $26 \%$ of cases.

Thus in comparison with the two largest series of patients with tamponade ${ }^{210}$ our group of patients had fewer signs of tamponade-nevertheless they derived benefit from pericardiocentesis.

ECHOCARDIOGRAPHIC SIGNS IN PATIENTS WITH SUSPECTED TAMPONADE

Chamber collapse alone was an unreliable sign of the presence of tamponade as features of chamber collapse did not occur in $29 \%$ of patients in whom pericardiocentesis relieved dyspnoea.

Right atrial collapse was found in $42 \%$ patients with tamponade-a value much lower than in other series $(92-100 \%) .{ }^{510}$ Despite the relatively low pressure in the right atrium compared with that in the right ventricle right atrial collapse was not found in seven of 16 echocardiograms where right ventricular collapse was present. This may reflect the difficulty in visualisation of the right atrium, adherence of the right atrium to the chest wall after cardiac surgery ${ }^{3}$ or the more rigorous definition of atrial collapse compared with that of ventricular collapse in our study.

Right ventricular collapse is often used as the main echocardiographic marker for tamponade; however, this was found in only $62 \%$ of our cases obtaining symptomatic relief 
from pericardiocentesis compared with $57 \%$ in the series comprising 50 patients of Levine $e t$ al. ${ }^{10}$ Previous simultaneous haemodynamic and two-dimensional echocardiographic measurements in patients undergoing pericardiocentesis have shown that haemodynamic improvement first occurs at the point of disappearance of right ventricular diastolic collapse. ${ }^{7}$ This is followed by further improvement in cardiac output and subsequent disappearance of right atrial collapse during pericardiocentesis.

The development of right ventricular collapse is dependent on right ventricular pressure, determined by the intravascular volume and pulmonary artery pressure, being less than pericardial pressure. ${ }^{11} 12$ While right ventricular collapse may be present, however, it does not necessarily indicate that the clinical features of tamponade are present; a study of 187 patients with pericardial effusions of all sizes demonstrated that over one half of patients with evidence of right ventricular collapse did not have cardiac tamponade at the time of echocardiography, nor did they subsequently develop this condition. ${ }^{13}$

The left atrium was the most difficult chamber to visualise (not clearly visible to both observers in $24 \%$ of all cases of pericardial aspiration) and left atrial collapse was infrequently associated with tamponade both in our series $(3 / 21$ patients $(19 \%))$ and in others. ${ }^{310}$

Left ventricular diastolic collapse was seen only in one patient who developed localised tamponade 12 months after cardiac surgery. Localised tamponade is more frequent postcardiac surgery and the classical echocardiographic features of right sided chamber collapse may not be present because the fluid tends to collect posterolaterally. ${ }^{3}$ The presence of left ventricular collapse is rare in nonsurgical patients; being absent in 50 consecutive patients with tamponade. ${ }^{10}$

Eisenberg et al $^{13}$ found that the main factor determining whether pericardial tamponade develops in patients with pericardial effusion is the size of the effusion; however, nine (43\%) of 21 of our patients with tamponade had moderate or small effusions. Thus size alone is not a sensitive indicator of tamponade.

Cardiac tamponade is therefore often associated with evidence of chamber collapse, however, its absence does not exclude tamponade.

\section{DETERMINANTS OF SUCCESSFUL PERICARDIAL ASPIRATION}

How much fluid needs to be aspirated to give symptomatic relief from an effusion in patients with cardiac tamponade? Experimental work in dogs has indicated that intrapericardial pressure is not linearly related to the volume of pericardial effusion. ${ }^{14}$ As the pericardial volume reaches its maximum small increments in volume cause large increments in pericardial pressure. ${ }^{14}$ The volume of pericardial fluid required to improve symptoms of tamponade therefore probably varies from patient to patient depending on the position on the pericardial pressure-volume curve. We found that dyspnoea was relieved in all patients with pericardial aspirate volumes
$>120 \mathrm{ml}$ but not in those with volumes $<120 \mathrm{ml}$.

The only factor associated with failure to drain an effusion was the presence of loculation. The development of loculation may relate to the length of time that the effusion has been present; one effusion while initially non-loculated and successfully aspirated recurred and became loculated over a period of three weeks and could not be aspirated. Similar progression of non-loculated to loculated effusions after pericardiocentesis has been noted by $\mathrm{Hsu}$ et al. ${ }^{6}$ Another factor predisposing to loculation may be pericardial trauma as Chuttani et $a l^{3}$ found loculated effusions in 15 of 18 pericardial effusions causing tamponade after cardiac surgery. This was not the case in our series in which the proportion of loculated effusions was similar irrespective of whether or not the patient had undergone cardiac surgery. While pericardiocentesis was difficult in patients with pericardial loculation, it was not always impossible; over 11 of fluid was aspirated from one patient with a loculated effusion. The presence of loculation should therefore not be viewed as an absolute contraindication to pericardiocentesis.

\section{Conclusion}

Our study demonstrates that no single physical or echocardiographic sign can be relied on as a marker of a pericardial effusion causing symptoms. The complete absence of physical or echocardiographic signs of tamponade does not exclude symptomatic benefit being derived from pericardial aspiration. Pericardial aspiration is safe in appropriate hands, although aspiration of loculated effusions may not be as successful as aspiration of non-loculated effusions.

1 Starling EH. Some points in the pathology of heart disease. Lancet 1897;1:652-5.

2 Guberman B, Fowler N, Engel P, et al. Cardiac tamponade in medical patients. Circulation 1981;64:633-40.

3 Chuttani K, Pandian N, Mohanty P, et al. Left ventricular diastolic collapse. An echocardiographic sign of regional cardiac tamponade. Circulation 1991;83:1999-2006.

4 D'Cruz IA, Cambell C, et al. Two dimensional echocardiography in cardiac tamponade occurring after cardiac surgery. $₹$ Am Coll Cardiol 1985;5:1250-2.

5 Gillam L, Guyer D, Gibson T, et al. Hydrodynamic compression of the right atrium: a new echocardiographic sign of cardiac tamponade. Circulation 1983;68:294-301.

$6 \mathrm{Hsu}$ T, Chen C, Hsiung M, et al. Paradoxical motion of the left ventricular free wall in cardiac tamponade. $A m$ Heart $\mathcal{F}$ 1986;111:807-8.

7 Singh S, Wann L, Schuchard G, et al. Right ventricular and right atrial collapse in patients with cardiac tamponade: a combined echocardiographic and hemodynamic study. Circulation 1984;70:966-71.

8 Fowler N. Cardiac tamponade. A clinical or an echocardiographic diagnosis? Circulation 1993;87(5):1738-41.

9 Tunick PA, Nachamie M, Kronzon I. Reversal of echocardick PA sions of pericardial tamponade by echocardiographic signs of pericardial tamponad
sion. Am Heart $₹$ 1990;119(1):199-201.

10 Levine MJ, Lorell BH, Diver DJ. Implications of echocardiographically assisted diagnosis of pericardial tamponade in contemporary medical patients: detection before ade in contemporary medical patients: detection before 17(1):59-65.

11 Leimgruber $\mathrm{P}$, Klopfenstein $\mathrm{H}$, Wann $\mathrm{L}$, et al. The hemodynamic derangement associated with right ventricular dynamic derangement associated with right ventricular echocardiographic study. Circulation 1983;68:612-20.

12 Klopfenstein HS, Cogswell TL, Bernath GA, et al. Alterations in intravascular volume affect the relation Alterations in intravascular volume affect the relation between right ventricular diastolic collapse and hemodynamic severity of

13 Eisenberg M, Oken K, Guerrero S, et al. Prognostic value of echocardiography in hospitalized patients with pericardial effusion. Am $\mathcal{F}$ Cardiol 1992;70:934-9.

14 Millard R, Fowler N, Gabel M. Hemodynamic and regional blood flow distribution responses to dextran, hydrallazine, isoprenaline, and amrinone during experimental cardiac tamponade. $\mathcal{F}$ Am Coll Cardiol 1983;1:
461-70. 